\title{
ABPP-HT* - Deep meets fast for activity-based profiling of deubiquitylating enzymes using advanced DIA mass spectrometry methods
}

\author{
Hannah B. L. Jones ${ }^{1{ }^{* *}}$, Raphael Heilig ${ }^{1 \sharp}$, Simon Davis ${ }^{2,1}$, Roman Fischer ${ }^{1,2}$, Benedikt M. Kessler \\ 1,2,*, and Adán Pinto-Fernández 1,2,*
}

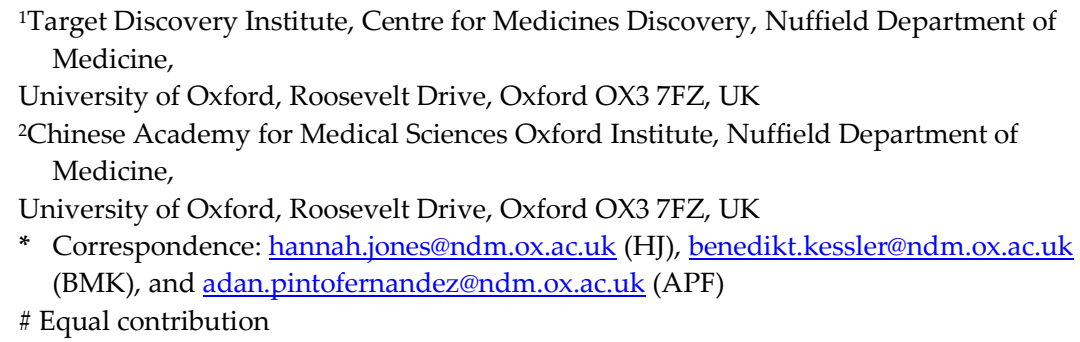

\begin{abstract}
:
Activity-based protein profiling (ABPP) uses a combination of activity-based chemical probes with mass spectrometry (MS) to selectively characterise a particular enzyme or enzyme class. ABPP has proven invaluable for profiling enzymatic inhibitors in drug discovery. When applied to cell extracts and cells, challenging the ABP-enzyme complex formation with a small molecule can simultaneously inform on potency, selectivity, reversibility/binding affinity, permeability, and stability. ABPP can also be applied to pharmacodynamic studies to inform on cellular target engagement within specific organs when applied to in vivo models. Recently, we established separate high depth and high throughput ABPP (ABPP-HT) protocols for the profiling of deubiquitylating enzymes (DUBs). However, the combination of the two, deep and fast, in one method has been elusive. To further increase the sensitivity of the current ABPP-HT workflow we implemented state-of-the-art dataindependent acquisition (DIA) and data-dependent acquisition (DDA) MS analysis tools. Hereby, we describe an improved methodology, ABPP-HT* (enhanced high-throughput-compatible activity-based protein profiling), that in combination with DIA MS methods allowed for the consistent profiling of 35-40 DUBs and provided a reduced number of missing values, whilst maintaining a throughput of 100 samples per day.
\end{abstract}

Keywords: activitomics, activity-based probes, chemical biology, dataindependent acquisition mass spectrometry (DIA), deubiquitylating enzymes, drug discovery, mass spectrometry, proteomics, ubiquitin.

\section{Introduction}

Activity-based probes (ABPs) react with the active site of an enzyme to inform on its activity. Typically, they are formed of a warhead that binds irreversibly, a specificity motif to ensure selectivity for a particular enzyme or enzyme family, and a reporter tag [1-3]. Their specificity means that ABPs can be applied to monitor the activity of enzymes in a cellular environment without the need for enzyme purification. Depending on the ABP, they can be applied to intact cells, or used with cellular lysates directly [4,5]. ABPs are invaluable for profiling potential 
enzymatic inhibitors in the early stages of drug discovery [6,7] . The prevention of ABP-enzyme complex formation by a small molecule inhibitor can inform on multiple compound parameters such as potency and selectivity in lysates, reversibility/binding affinity, permeability and stability within intact cells. ABPs used in combination with pharmacodynamic studies can also inform on inhibitor target engagement within cells and specific organs in vivo.

A family of enzymes that are currently being targeted for therapeutic inhibition are deubiquitylating enzymes (DUBs). DUBs oppose the process of ubiquitination, a post-translational modification (PTM) of proteins, responsible for the regulation of numerous cellular functions, such as degradation and signaling [8,9]. In some cases, the removal of ubiquitin from specific protein targets by a DUB can prevent proteasomal proteolysis or degradation by the autophagosome/lysosome system [10]. Currently DUB inhibitors are being developed for therapeutic treatment of a number of diseases including Parkinson's disease and cancer [11].

Previously, we have accomplished in-depth profiling of DUB inhibitors using a combination of ABP immunoprecipitation and LC-MS/MS proteomic analysis, a methodology known as ABPP (activity-based protein profiling) [12]. With further improvements of this technique, we have demonstrated the ability of a ubiquitin-based $\mathrm{ABP}$ to form complexes with 74 endogenous DUBs in MCF-7 breast cancer cells [13]. These 74 DUBS represented the majority of active cysteine-protease DUBs, including distinguishable isoforms, expressed in the human genome [13,14]. The Ub-based ABP used in this study is formed of a HA tag for immunoprecipitation, ubiquitin for specificity, and a propargylamine warhead for binding to the active site cysteine (HA-UbPA).

This in-depth profiling was achieved with low $\mathrm{pH}$ C18 HPLC prefractionation, resulting in 10 samples concatenated from 60 fractions that were subsequently analysed using a 60-minute gradient per fraction on an Orbitrap LC-MS/MS system [13]. While this in-depth ABPP would be particularly valuable for a late stage drug candidate, it does not offer the necessary throughput for compound screening in the early stages of drug discovery. Without the pre-fractionation of samples, a 60-minute orbitrap LC-MS/MS gradient typically leads to the identification and quantitation of $\sim 30-40$ DUBs $[15,16]$. Whilst the reduced number of DUBs identified can indeed act as a representative panel of DUBs for drug screening, the throughput of around 10 samples per day is still limiting drug discovery applications.

To overcome this, we recently developed methodology that employed the use of the Agilent Bravo AssayMAP liquid handling platform to improve the $\mathrm{ABPP}^{\prime}$ 's immunoprecipitation and sample preparation throughput, and applied this in combination with runtimes of 15 minutes per sample on a Evosep/Bruker timsTOF LC-MS/MS [17]. This methodology, termed ABPP-HT, enabled a 10-fold increase in throughput, allowing for the analysis of 100 samples per day. However, the increase in throughput led to a reduced profiling depth, typically resulting in the identification and quantitation of $\sim 15-25$ DUBs. Although these DUBs can still be used as a representative panel for screening purposes, the decreased DUBome depth makes the methodology less suitable for low abundance DUBs and may mean key compound cross-reactivity data is overlooked.

In this work, we sought to improve the DUBome profiling depth whilst retaining the increased throughput offered by the Bravo/Evosep/timsTOF 
combination. This was achieved via the exploration of improved data acquisition and analysis methodology. Our previous methodology employed a widely used search engine software (MaxQuant) in combination with data-dependent acquisition (DDA) where the most abundant precursor ions are selected for further fragmentation. While powerful, this semi-stochastic precursor picking leads to missing values for low abundance peptides, resulting in data reproducibility issues and reduced sensitivity [18]. Data-independent acquisition (DIA) is advantageous as it selects all peptides within a given $\mathrm{m} / \mathrm{z}$ window, giving reproducible precursor ions, leading to fewer missing values. Due to the trapped ion mobility capabilities of the timsTOF, DIA data collection method diaPASEF (parallel accumulation-serial fragmentation combined with data-independent acquisition) provides the opportunity for sampling all peptide precursor ions, resulting in improved depth and reproducibility [19].

Recently there has been an increase in the availability of freely available proteomic software packages with DIA analysis capabilities, which work with in silico libraries, negating the time and cost associated with project specific libraries. Those that are free and are compatible with diaPASEF data format include DIA-NN [20,21], and Maxquant's MaxDIA [22]. Here, for data collected using the ABPP-HT methodology, we have performed an in-depth comparison of free proteomic software packages including Maxquant and Fragpipe [23-26] for DDA analysis and DIA-NN and maxDIA for DIA analysis [20,22] (Scheme 1). By applying DUB inhibitors with established potency/selectivity features we were able to examine the reproducibility, sensitivity and accuracy of each data acquisition and analysis method. Through this comparison, we found that the DUBome profiling depth of the ABPP-HT methodology could be improved to match that of the lower throughput methodology, in particular when combined with DIA/DIA-NN, whilst the data reproducibility and quantitative accuracy is maintained. We termed this methodology, enhanced high-throughput-compatible activity-based protein profiling $\left(\mathrm{ABPP}-\mathrm{HT}^{*}\right)$.

\section{Results}

2.1 ABPP-HT DUBome depth search engine comparison

With samples processed using the ABPP-HT methodology, differences in DUBome depth were evaluated with different proteomic software packages using data acquired by both DDA and DIA. In the case of DIA acquired data, library free mode was applied to maintain the highthroughput nature of the experimentation whilst reducing costs. Here, we examined data obtained using both with and without "Matchbetween-runs" (MBR), a feature that helps to minimize the intrinsic missing value issue in proteomics by identifying peptides by ID transfer with tandem mass spectra from aligned runs, based on $\mathrm{m} / \mathrm{z}$, charge state, retention time, and ion mobility (if available). We felt it important to evaluate data both with and without this feature as the size of our sample cohort is a variable that may alter the efficiency of MBR. It is of note that DIA-NN recommends the match between runs (MBR) setting where library-free mode is being applied, and this should be considered when interpreting this dataset. The samples used for these comparisons comprised of MCF-7 cell lysates that were probe labelled and immunoprecipitated by the HA tag of the HA-Ub-PA probe in triplicate using the high-throughput method of combining Agilent's liquid 
handling platform, Evosep liquid chromatography and a timsTOF mass spectrometer as outlined previously [17].

Figure $1 \mathrm{~A}$ is a direct comparison of the total number of DUBs identified by DIA-NN and maxDIA for DIA data, as well as Fragpipe and Maxquant for DDA-acquired data. In all cases, other than maxDIA, applying match between runs (MBR) increased the identification and quantification of DUBs by LC-MS/MS, where samples from immunoprecipitations analysed in triplicate were matched. However, MBR only resulted in the identification of 1-3 extra DUBs on average, demonstrating the tractability of the methodology regardless of sample number. For analysis with Fragpipe, we compared the difference between 1 and 2 ion quantitation. This quantitation by Fragpipe's ionquant represents the number of quantifiable ions required for protein quantification. Previously, 1 ion quantitation has been shown to have a comparable median CV to Maxquant's 2 peptide minimum quantitation [26]. An increased stringency of a 2 ion minimum for quantitation vs a 1 ion minimum resulted in a reduction in DUBome depth. It is worth noting that for 1 ion minimum quantitation, with or without MBR, samples, all identified DUBs had a CV of $<=20 \%$, suggesting that the increased depth attained with this reduced stringency is not negatively impacting the quantitative accuracy of the identified DUBome. From this dataset, we found that the application of both Maxquant in DDA mode and maxDIA DIA mode, resulted in a significantly reduced DUBome depth, with the number of reproducibly identified DUBs $(\mathrm{CV}<=0.2)$ reduced by approximately half as compared to those identified by DIA-NN, and approximately a third compared to those identified by Fragpipe. From this initial result, we ascertained that both Maxquant and maxDIA were not the optimal tools for the analysis of ABPP-HT data, and subsequent comparisons were performed using Fragpipe and DIA-NN.

Figure 1A demonstrates that for the same samples subjected to different acquisition modes, DIA data analysed with DIA-NN is able to quantify more DUBs on average when compared to DDA data analysed with Fragpipe. All DUBs identified by DIA-NN are common to Fragpipe, with DIA-NN identifying an additional 10 DUBs that are not quantified by Fragpipe (Figure 1B). The high levels of commonality in DUBs identified by both Fragpipe and DIA-NN gives further confidence that the additional depth achieved by both methods, when compared to Maxquant and maxDIA, represents accurately identified DUBs. To ascertain whether the additional DUBs identified by both methods are as a direct result of $\mathrm{HA}-\mathrm{Ub}-\mathrm{PA}$ enrichment, the HA-Ub-PA samples were compared to a no HA-Ub-PA control sample (Figure 1C and 1D). All DUBs quantified from the Fragpipe analysis (2 ion minimum quantitation with MBR) were enriched $>10$ fold (other than USP4) with the majority only present in samples with HA-Ub-PA, meaning the identification of these DUBs is likely attributable to $\mathrm{ABP}$ binding and subsequent enrichment (Figure 1C). Contrastingly, 50\% of the DUBs quantified by DIA-NN (MBR) had valid intensity values in samples both with and without HA-Ub-PA. However, and reassuringly, all DUBs, except for USP15, USP34, USP38, USP48 and OTUD7B, were enriched > 10-fold where HA-Ub-PA was present (Figure 1D). The increase in the number of DUBs present in both the no probe and probe labelled samples with DIA-NN analysis is likely attributable to the increased sensitivity associated with diaPASEF data when compared to DDA data [19], as demonstrated by the increase in the number of proteins quantified overall 
(Total number of identified proteins: DIA-NN $=912$, Fragpipe $=218$ ). These additional proteins including the DUBs detected in the no probe sample will be sourced from co-immunprecipitation of protein complexes as well as non-specific background binding of proteins to the antibody and beads during the immunoprecipitation. However, it is important to note that in the presence of competitive $\mathrm{HA}-\mathrm{Ub}-\mathrm{PA}$ binding to the antiHA antibody, these background proteins may not be present in the sample elution at all. Whilst a no ABP control is important to ascertain which DUBs could potentially bind as background, these DUBs should still be considered as cross-reactive to an inhibitor if they are immunoprecipitated in a concentration-dependent manner. Therefore, absolute quantitation of these DUBs for the identification of $\mathrm{IC}_{50}$ values should be treated with caution and, in some cases, normalised to a no probe condition.

\subsection{Reproducibility and repeatability of Fragpipe and DIA-NN for ABPP-} HT data

To ensure that the additional DUBome depth attained with both Fragpipe and DIA-NN was reproducible, we used the same probe labelled samples in triplicate to examine the number of unique peptides identified per DUB (Figure 2A), the coefficient of variation for the DUBome (Figure 2B), and the number of missing values within the DUBome (Figure 2C). The number of unique peptides identified per DUB followed the same trend regardless of whether Fragpipe (DDA) or DIANN (DIA) were used, or the settings applied, giving further confidence that the additional depth achieved by the data acquisition methods and software's is not artifactual. The increased sensitivity of DIA data vs DDA data is again clear, with DIA-NN consistently identifying more peptides than Fragpipe. DUBs that are identified by DIA-NN and not Fragpipe have a low number of peptides, which demonstrates that they are present as a result of the increased sensitivity of the DIA-NN/DIA combination.

Reproducibility of the DUBome, visualized as CVs [\%] at Figure 2B, shows that both DIA/DIA-NN and DDA/Fragpipe average low CVs of less than $10 \%$. Here, DIA-NN has slightly higher CVs overall when compared to Fragpipe. DIA-NN with and without MBR has some extreme outliers ( $\mathrm{CV}>40 \%$ ). The 2 ion minimum quantification for Fragpipe results is the most reproducible setting with CVs $<20 \%$, but at the cost of a reduced DUBome depth.

For the ABPP-HT approach, missing values present a considerable issue, as the absence of a DUB at a high inhibitor concentration may be indicative of complete inhibition, or of a missing value. This issue can be overcome by increasing the number of replicates. However, more replicates mean a lower throughput. This issue has been outlined previously in the context of stochastic DDA IP data [27], and so was considered here for both DIA-NN and Fragpipe analyses. Missing values are extremely low for both DIA-NN and Fragpipe, thereby not presenting an issue for this dataset (Figure 2C). Fragpipe and DIA-NN with MBR had no missing values at all, which is expected given that the MBR feature minimizes missing values across runs. The DUBs that did have 1 or 2 missing values for both Fragpipe and DIA-NN were quantified from 2 or less peptides. Inhibition of DUBs with low intensities that have been quantified from 2 or less peptides should be carefully considered in any case. 
2.3 Concentration-dependent quantification sensitivity, precision, and accuracy with DDA and DIA

To further test the sensitivity of DIA/DIA-NN and DDA/Fragpipe, we performed a titration of peptide injections on the timsTOF after immunoprecipitation of $100 \mu \mathrm{g}$ of lysate. Without MBR both DIA-NN and Fragpipe were able to detect a representative panel of 10-15 DUBs with only $50 \mathrm{ng}$ of peptides injected. For both the number of DUBs identified, and the number of proteins detected overall, DIA data with DIA-NN MBR was the most sensitive analysis across the titration (Figure 3A and 3B).

With confidence in the DUBome depth, reproducibility and sensitivity offered by both DIA/DIA-NN (MBR), and DDA/Fragpipe (MBR 2 ion minimum quant) we applied both workflows to specific and pan DUB inhibitors to check that the quantitation of the methods agree with each other in the context of DUB inhibition. Inhibition can be quantified by taking the intensity of a DUB immunoprecipitated by the HA-Ub-PA probe in the presence of an inhibitor and normalizing it to a control with no inhibitor present. A concentration dependence, for USP7, with the pan DUB inhibitor PR619 resulted in small differences in quantitation between DDA/Fragpipe quantitation and DIA/DIA-NN quantitation (Figure 3C). Both data sets stray from the expected fit with the same trend (Figure 3D), which may be indicative of the complex kinetics associated with a pan-DUB inhibitor and subsequent HA-Ub-PA binding. Complementing this data, a concentration dependence with the USP7 specific inhibitor FT827 resulted in good agreement between the DDA/Fragpipe and the DIA/DIA-NN methodology (Supplementary Figures 1A, 1B, 1D and 1E).

Quantitation accuracy was further validated by evaluating the difference in remaining activity between DDA/Fragpipe data and DIA/DIA-NN data for DUBs that were common to both datasets for inhibitors PR619 (Figure 3E) and FT827 (Supplementary Figure 1C). Although there is variability between the Fragpipe and DIA-NN datasets, the variability does not occur in a concentration dependent manner, meaning that the selectivity of FT827, and the pan-reactivity of PR619, aligns across the two datasets.

\subsection{Applicability of ABPP-HT* to DUB inhibitor screening}

FT827 is a highly selective USP7 inhibitor, HBX41108 is a cross-reactive USP7 inhibitor, P22077 is a selective USP7 inhibitor with known crossreactivity to USP47, and PR619 is a pan-DUB inhibitor [12,17,28]. As a proof-of-concept experiment, inhibitor profiles were assessed across the identified DUBomes by DDA/Fragpipe and DIA/DIA-NN. Four DUBs at the low end of the LC/MS-MS dynamic range were discarded from the DIA/DIA-NN dataset as they did not show a concentration dependent profile, most likely due to inaccurate quantification at lower abundance. Despite this, DIA/DIA-NN continued to provide an increased sensitivity, with the identification of 29 DUBs, compared to 22 DUBs identified by Fragpipe (Figure 4A).

Both DIA/DIA-NN and DDA/Fragpipe confirmed the previously described inhibitor profiles in their quantitation across the DUBome (Figure 4A). One exception is that Fragpipe does not confirm USP47 inhibition in response to P22077 treatment, as previously described $[12,29]$. 
Although missing values were found to be comparable between DIA/DIA-NN and DDA/Fragpipe with probe labelled samples in triplicate (Figure 2C), when applied across a larger sample cohort, DIA/DIA-NN displays fewer missing values compared to DDA/Fragpipe (Figure 4A). This is especially apparent at high concentrations of PR619 treatment across the DUBome. The quantitation of these values allows for the conclusion that a value is reduced as a consequence of inhibitor treatment, rather than it being missing perhaps as a consequence of inhibitor treatment, or perhaps as a consequence of missing values occurring due to the stochastic nature of DDA LC-MS/MS.

IC50 values for all the tested USP7 DUB inhibitors were calculated (Figure 4B). It is of note that HBX41108 and P22077 were also assessed for quantitation overlap, although neither inhibitor is potent enough to lead to accurate $\mathrm{IC}_{50}$ values, resulting in large $95 \%$ confidence intervals. For FT827, HBX41108 and P22077 the $95 \%$ confidence intervals overlap for Fragpipe, DIA-NN and previously published Maxquant data (Figure 4B). For PR619, the USP7 IC 50 value and $95 \%$ confidence intervals align between DIA-NN and Maxquant, but do not overlap with Fragpipe. This combined with the overall smaller $95 \%$ confidence intervals and higher $\mathrm{R}$ squared values of DIA-NN compared to Fragpipe suggests that the quantitation from the DIA/DIA-NN combination may be more accurate.

\section{Discussion}

The potency and selectivity of an inhibitor in the cellular environment can be highly affected by limited permeability, degradation, and reactivity with off-target events. When a suitable activity-based probe is available, ABPP assays can inform on how the inhibitor is engaging its target in a cellular matrix.

One of the classical limitations of the ABPP assay was its intrinsic low throughput due to probe affinity purifications being to be performed by hand. This is particularly prevalent when applying this assay for small molecule inhibitor screening. We have recently developed a highthroughput compatible activity-based protein profiling (ABPP-HT) that allows the semi-automated analysis of multiple samples in a microplate format. To achieve this, we automated the affinity purification and proteomic sample preparation steps via a liquid handling robot combined with the high-throughout-compatible LC-MS/MS platform Evosep/tims TOF [17].

While ABPP-HT increased the throughput of the traditional ABPP ten times approximately, the proteomic depth was significantly reduced. Around 15-25 DUBs were identified with this methodology, clearly below the numbers of the classical ABPP ( 30-40 DUBs IDs;[30]) and significantly lower than our previously reported high-depth ABPP $(>70$ DUB IDs;[13]).

To improve sensitivity and proteomic depth covered by the ABPPHT workflow we decided to test DIA-NN, and maxDIA for dataindependent acquisition method mass-spectrometry (DIA) [20,22]. DIA allows the quantitation of low abundance peptides and overcomes the intrinsic missing values limitation of DDA, due to the stochastic nature of top- $\mathrm{N}$ fragmentation [31]. Recently, improved DIA search engines have been developed, resulting in substantially improved coverage in complex proteomics phosphoproteomics [32] and ubiquitomics workflows [33,34]. We also compared Fragpipe, a more recently 
developed proteomics search engine for data-dependent acquisition mass-spectrometry (DDA), against the existing DDA software MaxQuant [23-26].

When using DIA/DIA-NN, the number of identified DUBs went up to 38 DUBs (matching between runs) in a comparable batch of samples. This is a considerable improvement ( $50 \%)$ over using MaxQuant combined with DDA. When implementing the DDA search engine Fragpipe we were able to consistently identify up to 28 DUBs. We also tested MaxDIA, the software platform for analyzing library-free DIA data using the MaxQuant environment [22]. For ABPP-HT, both MaxQuant and MaxDIA showed less coverage as compared to DIA-NN and Fragpipe so we decided to base our comparisons with these two search engines, reflecting for DDA and DIA based analysis pipelines.

In terms of reproducibility and repeatability both Fragpipe and DIANN performed comparably when the number of samples was low, as shown by the low coefficients of variation and the near absence of missing values, with or without MBR. However, DIA-NN MBR was consistently more sensitive than Fragpipe when injecting increasing amounts of peptides coming from $100 \mu \mathrm{g}$ of protein starting material.

Finally, we profiled a panel of USP7 small molecule inhibitors P22077 [12], HBX41108 [35] and FT827 [28] and pan-DUB inhibitor PR619 [12] using our ABPP-HT* (enhanced high-throughput-compatible activity-based protein profiling). Both search engines managed to provide comparable dose-dependent inhibition profiles for USP7 in lysates treated with a range of inhibitor concentrations. Selectivity profiles for all inhibitors led to expected DUBome reactivity, with FT827 displaying high specificity for USP7, and PR619 displaying high levels of reactivity across a representative panel of 22 (Fragpipe) and 29 (DIA-NN) DUBs, respectively. It is important to note, that when analyzing a larger number of samples, DIA-NN not only displays more sensitivity but also a significant reduction in the number of missing values when compared to Fragpipe/DDA. This is highly relevant for this application as it can lead to false positives and, therefore, flawed inhibition and cross-reactivity profiles.

In summary, by optimizing the data acquisition and the search engine we managed to increase proteomic and DUBome depth of our current high-throughput methodology by a $\sim 50 \%$, allowing for a fast and deep profiling of representative cellular cysteine peptidase DUBs (Scheme 2). This is particularly interesting for drug discovery efforts as it allows for the acquisition of target engagement data (i.e. potency and selectivity) in a cellular context. Another potential application is the DUB profiling of a relatively large cohort sample, suitable for clinical proteomics studies.

\section{Materials and Methods}

\subsection{Cell culture and lysis}

MCF-7 cells (ATCC HTB-22) were cultured and lysed as previously described [17]. Briefly, cells were cultured in high glucose Dulbecco's Modified Eagle's Medium (DMEM) with 10 \% Fetal Bovine Serum, at 37 ${ }^{\circ} \mathrm{C}, 5 \% \mathrm{CO}_{2}$. Cells were washed and scraped in phosphate-buffered saline (PBS), and collected at $300 x g$. Cells were lysed in $50 \mathrm{mM}$ Tris Base, $5 \mathrm{mM}$ $\mathrm{MgCl} 2 \cdot 6 \mathrm{H} 2 \mathrm{O}, 0.5 \mathrm{mM}$ EDTA, $250 \mathrm{mM}$ Sucrose and $1 \mathrm{mM}$ Dithiothreitol 
(DTT) (pH 7.5). Lysis was carried out through $10 \times 30$ second vortexing of the lysate using an equal volume of acid washed glass beads, with 2 minutes breaks on ice. Lysates were clarified through centrifugation at $14,000 x g$, at $4{ }^{\circ} \mathrm{C}$ for 25 minutes.

\subsection{HA-Ub-PA synthesis}

HA-Ub-PA was synthesized as previously described $[13,17,36]$. HAUbiquitin (Gly76del)-intein-chitin binding domain (CBD) was expressed in E. coli. The cell pellet was suspended in $50 \mathrm{mM}$ Hepes, $150 \mathrm{mM} \mathrm{NaCL}$, $0.5 \mathrm{mM}$ DTT and sonicated for 30 seconds X 10, with 30 second breaks. The lysate was purified using Chitin bead slurry, and incubated with 100 $\mathrm{mM}$ MesNa overnight at $37^{\circ} \mathrm{C}$ to form HA-Ub-MesNa. Incubation with $250 \mathrm{mM}$ propargylamine (PA) at room temperature for 20 minutes, followed by PD-10 desalting resulted in HA-Ub-PA formation, as confirmed by western blot and intact protein LC-MS (data not shown).

\subsection{HA-Ub-PA and inhibitor labelling}

Lysates were diluted to a $3.33 \mathrm{mg} / \mathrm{ml}$ protein concentration, with 250 $\mu \mathrm{g}$ of protein per reaction unless otherwise stated (accounting for dilution with HA-Ub-PA and inhibitor). Lysate was incubated with inhibitors (or DMSO for the control) for $1 \mathrm{hr}$ at $37^{\circ} \mathrm{C}$. HA-Ub-PA was then incubated with lysate with/without inhibitor labelling for 45 minutes at $37{ }^{\circ} \mathrm{C}$. Reactions were quenched using NP40 (0.5 \% v/v) and SDS (0.5 \% w/v), and diluted to $1 \mathrm{mg} / \mathrm{ml}$ lysate protein concentration using NP40 buffer (50 mM Tris, 0.5 \% NP40 (v/v), 150 mM NaCL, 20 mM MgCl2, pH 7.4).

\subsection{Agilient Bravo Assay MAP Liquid handling platform} immunoprecipitation

The immunoprecipitation methodology used in this paper is as outlined previously using the original ABPP-HT methodology [17]. Briefly, $100 \mu \mathrm{g}$ of anti-HA antibody (12CA5) was immobilized on Protein A cartridges (Agilent, G5496-60000), using the in-built immobilization methodology, with PBS used for all wash steps. The in-built affinity purification methodology was used for HA-Ub-PA immunoprecipitation, with standard settings other than a slow flow-rate for lysate loading $(1 \mu \mathrm{l} / \mathrm{min})$ to ensure optimal antibody binding. Peptides were eluted with $50 \mu \mathrm{l}$ of $0.15 \%$ TFA.

\subsection{Mass spectrometry sample preparation}

Samples were neutralized with $180 \mu \mathrm{l}$ of $100 \mathrm{mM}$ TEAB, pH 8.5, and digested overnight at $37{ }^{\circ} \mathrm{C}$ with $1 \mu \mathrm{g}$ of trypsin (Worthington, LS003740 TPCK-treated). Samples were then acidified with formic acid ( $1 \%$ final concentration).

\subsection{Evosep/timsTOF LC-MS/MS}

Solvent A was $0.1 \%$ formic acid in water and Solvent B was $0.1 \%$ formic acid in acetonitrile. All centrifugation steps were $700 \mathrm{~g}$ for 60 seconds unless stated otherwise. For peptide loading onto EvoTips (Evosep) [37], tips were first activated by soaking in 1-propanol, then washed with $20 \mu \mathrm{L}$ of Solvent B by centrifugation. Washed tips were conditioned by soaking in 1-propanol until the C18 material appeared pale white. Conditioned tips were equilibrated by centrifugation with 20 $\mu \mathrm{L}$ Solvent A. Samples were then loaded into the tips while the tips were soaking in Solvent A to prevent drying, peptides were then bound to the 
C18 material by centrifugation. Tips were washed by centrifuging with $20 \mu \mathrm{L}$ Solvent A. Next, $100 \mu \mathrm{L}$ Solvent A was added to the tips and the tips were centrifuged at $700 \mathrm{xg}$ for 10 seconds. Samples were then immediately analysed by LC-MS/MS.

Peptides were analysed using an Evosep One (Evosep) [37] coupled to a timsTOF Pro mass spectrometer (Bruker) using a $100 \mu \mathrm{m} \times 80 \mathrm{~mm}$ C18 column packed with $3 \mu \mathrm{m}$ beads (PepSep, EV-1109). The pre-set "100 samples per day" method was used, resulting in a gradient length of 11.5 minutes at a flow rate of $1.5 \mu \mathrm{L} / \mathrm{min}$. The timsTOF Pro was operated in parallel accumulation, serial fragmentation (PASEF) mode. TIMS ion accumulation and ramp times were set to $100 \mathrm{~ms}$ and mass spectra were recorded from $100-1700 \mathrm{~m} / \mathrm{z}$.

\subsection{Data-dependent acquisition methods}

The ion mobility range was set to $0.85-1.30 \mathrm{Vs} / \mathrm{cm}^{2}$. Precursor ions selected for fragmentation were isolated with an ion mobility-dependant collision energy that increased linearly from $27-45 \mathrm{eV}$ over the ion mobility range. Three PASEF MS/MS scans were collected per full TIMSMS scan, giving a duty cycle of $0.53 \mathrm{~s}$. Ions were included in the PASEF MS/MS scans if they met an intensity threshold of 2000 and were sampled multiple times until a summed target intensity of 10000, once sampled, ions were excluded from reanalysis for 24 seconds.

\subsection{Data-independent acquisition methods}

The mass spectrometer was operated in diaPASEF mode using 8 diaPASEF scans per TIMS-MS scan, giving a duty cycle of $0.96 \mathrm{~s}$ [19].

For Figures 1, 2, 3C-3E and 4 the ion mobility range was set to $0.6-$ $1.6 \mathrm{Vs} / \mathrm{cm}^{2}$. Each mass window isolated was $25 \mathrm{~m} / \mathrm{z}$ wide, ranging from $400-1000 \mathrm{~m} / \mathrm{z}$ with an ion mobility-dependent collision energy that increased linearly from $20 \mathrm{eV}$ to $59 \mathrm{eV}$ between $0.6-1.6 \mathrm{Vs} / \mathrm{cm}^{2}$ (Table S1).

Due to a software upgrade, for Figure $3 \mathrm{~A}+\mathrm{B}$ the ion mobility range was set to $0.85-1.3 \mathrm{Vs} / \mathrm{cm}^{2}$. Each mass window isolated was $25 \mathrm{~m} / \mathrm{z}$ wide, ranging from $475-1000 \mathrm{~m} / \mathrm{z}$ with an ion mobility-dependent collision energy that increased linearly from $27 \mathrm{eV}$ to $45 \mathrm{eV}$ between $0.85-1.3$ $\mathrm{Vs} / \mathrm{cm}^{2}$ (Table S2).

\subsection{Software settings}

All software was set to default settings unless stated (e.g. MBR vs no MBR), with N-terminal acetylation and Methionine oxidation set as variable modification, and no fixed modifications. All searches used Homo sapiens Uniprot database (retrieved 16-04-2021), other than maxDIA which requires its own generated FASTA (UP000005640_9606). Software versions: Fragpipe 17.1 (MSFragger 3.4, Philosopher 4.1.1, Python 3.9.7). DIA-NN 1.8, Maxquant 2.0.3.

\subsection{Data analysis}

Graphs were generated and fitted using Graphpad prism 9.2.0 (333), other than the upset plot (Figure 1B) [38]. For Fragpipe/Maxquant/maxDIA unique/razor 'maxLFQ' intensities were used, for DIA-NN razor intensities are not assigned to a protein group and so unique intensities were used for Figures 1-3 to avoid overestimating the number of DUBs present, and for Figures 3A and 3B to avoid counting the same protein multiple times. Figures 1D and 4 
includes DIA-NN razor intensities which are denoted as DUB1;DUB2. Unique Fragpipe peptides were counted from the output file 'protein.tsv'. Proteotypic DIA-NN peptides with intensities $>0$ were extracted from the output file report.pr_matrix.tsv, with precursors averaged to give unique peptide numbers.

Supplementary Materials: Figure S1: USP7 FT827 DIA-NN/Fragpipe quantitation comparison, Table S1: DIA-PASEF windows for data in Figures 1, 2 ,3C - E + 4, Table S2: DIA-PASEF windows for data in Figures 3A + B.

Author Contributions: HJ, RF, BK, and AP-F directed this study. Most experiments were devised by HJ, RH, BK, and AP-F and carried out by $\mathrm{HJ}, \mathrm{RH}$, and AP-F. HJ, BK, SD and AP-F wrote the paper. All authors commented on the text.

Funding: B.M.K. and A.P.-F. were supported by the Chinese Academy of Medical Sciences (CAMS) Innovation Fund for Medical Science (CIFMS), China (grant number: 2018-I2M-2-002) and by Pfizer. H.B.L.J. was supported by a BrystolMeyers Sqibb award (B.M.K).

Data Availability Statement: Datasets have been submitted to ProteomeXchange via PRIDE (PE accession number will be added once the submission is complete).

Acknowledgments: We would like to thank the Discovery Proteomics Facility (led by Roman Fischer and Iolanda Vendrell) at the Target Discovery Institute (Oxford) for expert help with the analysis by mass spectrometry.

Conflicts of Interest: The authors declare that the research was conducted in the absence of any commercial or financial relationships that could be construed as a potential conflict of interest.

The authors declare that this study received funding from Bristol Myers Squibb, Pfizer Inc. and the Chinese Academy of Medical Sciences. The funders were not involved in the study design, collection, analysis, interpretation of data, the writing of this article or the decision to submit it for publication.

Figure legends

Scheme 1. Data collection and analysis comparisons for the development of the ABPP-HT* approach to improve the DUBome depth while maintaining the increased throughput gained through the implementation of a liquid handling platform (Bravo, Agilent) in combination with a timsTOF LC-MS/MS (Evosep/Bruker) for ABPP assays.

Figure 1. A) ABPP-HT DUBome depth search engine comparison. Bars from left to right: Mean number of DUBs identified and quantified by each software across three replicate IPs (+/- SD), number of DUBs that have three values across the replicates, and number of DUB intensities with $\mathrm{CVs}<20 \%$. CVs exclude missing values of 0 for calculation. If a DUB is only identified in 1 replicate then the $\mathrm{CV}$ is counted as $>20 \%$. B) Upset plot of the distribution of identified DUBs across the different data collection and analysis methods. C) Enrichment of DUBs with DDA data analysed with Fragpipe MBR 2 ion minimum quant and D) DIA-NN MBR when compared to a sample with no HA-Ub-PA. HA-Ub-PA n=3, No HA-Ub-PA $\mathrm{n}=1$. Samples were Log2 transformed, averaged, and missing values imputed at a set value of 0.5 .

Figure 2. A) Reproducibility and repeatability of Fragpipe and DIA-NN for ABPP-HT data. Mean number of peptides from quantified DUBs. For all DIA-NN searches proteotypic peptides with intensities $>0$ were counted. For all Fragpipe searches unique peptides with intensities $>0$ were counted. B) CVs for DUBs quantified from DIA-NN and Fragpipe searches, upper and lower whisker limits represent CVs within the $10-90^{\text {th }}$ percentiles. Outliers denoted as symbols. C) Number of missing values for DUBs quantified from DIA-NN and Fragpipe searches. 
bioRxiv preprint doi: https://doi.org/10.1101/2022.02.18.480987; this version posted February 21,2022 . The copyright holder for this preprint (which was not certified by peer review) is the author/funder, who has granted bioRxiv a license to display the preprint in perpetuity. It is made available under aCC-BY-ND 4.0 International license.

Figure 3. A-D) Concentration-dependent quantification precision and sensitivity with DDA and DIA. Increasing quantities of peptides injected into the timsTOF from an IP with $100 \mu \mathrm{g}$ of lysate protein starting material. A) Number of quantified proteins for DIA-NN and Fragpipe 1 ion quant $(+/-\mathrm{MBR})$. B) Number of quantified DUBs for DIA-NN and Fragpipe 2 ion quant (+/- MBR). C) USP7 IC50 curve showing reduction of USP7 intensity with increasing PR619 concentrations, collected as either DIA data analysed with DIA-NN MBR* or DDA data Fragpipe data 2 ion quant $\mathrm{MBR}^{*}$ fit to $\mathrm{Y}=100 /\left(1+10^{\wedge}((\mathrm{X}-\log \mathrm{IC} 50))\right)$. ${ }^{*} \mathrm{MBR}$ as part of a larger dataset with other inhibitors. D) Predicted vs actual $\mathrm{Y}$ values of the $\mathrm{IC}_{50}$ curve shown in C). E) Difference plot of common DUBs identified by both DIA-NN MBR and Fragpipe 2 ion quant MBR with DIA-NN \% activity subtracted from Fragpipe \% activity at various PR619 concentrations.

Figure 4. Applicability of ABPP-HT* to DUB inhibitor screening. A) Heatmap showing the \% remaining activity of a panel of DUBs quantified from DDA data with Fragpipe 2 ion quant MBR (green), and DIA-NN MBR (red) at various concentrations of USP7 inhibitors (FT827, HBX41108, P22077) and a pan DUB inhibitor (PR619). DUBs that did not contain valid values across all three control replicates were discounted. DUBs that were not enriched by an order of magnitude when compared to a no HA-Ub-PA control were also discounted. The 4 DUBs with the lowest intensities (USP35, USP45, USP1 + ATXN3) did not behave in an inhibitor concentration dependent manner for DIA-NN and were discounted from the analysis. B) Table to show $\mathrm{IC}_{50}$ s values with $95 \%$ confidence interval (CI) values for inhibitors fit to $Y=100 /\left(1+10^{\wedge}\left(\left(X-\log I C_{50}\right)\right)\right)$.

Scheme 2. A comparison of the increased throughput and DUBome depth achieved by the ABPP-HT* when compared to the original ABPP-HT methodology. ABPP-HT* enables higher throughput than immunoblotting, traditional benchtop ABPP immunoprecipitations and fractionated ABPPs, whilst maintaining a depth to allow for the identification of a representative panel of DUBs.

\section{References}

1. Chen, X.; Wong, Y.K.; Wang, J.; Zhang, J.; Lee, Y.-M.; Shen, H.-M.; Lin, Q.; Hua, Z.-C. Target Identification with Quantitative Activity Based Protein Profiling (ABPP). PROTEOMICS 2017, 17, 1600212, doi:10.1002/pmic.201600212.

2. Chakrabarty, S.; Kahler, J.P.; van de Plassche, M.A.T.; Vanhoutte, R.; Verhelst, S.H.L. Recent Advances in Activity-Based Protein Profiling of Proteases. In Current Topics in Microbiology and Immunology; Springer Verlag, 2018; Vol. 420, pp. 253-281.

3. Deng, H.; Lei, Q.; Wu, Y.; He, Y.; Li, W. Activity-Based Protein Profiling: Recent Advances in Medicinal Chemistry. European Journal of Medicinal Chemistry 2020, 191, 112151, doi:10.1016/j.ejmech.2020.112151.

4. Yee, M.; Fas, S.C.; Stohlmeyer, M.M.; Wandless, T.J.; Cimprich, K.A. A Cell-Permeable, Activity-Based Probe for Protein and Lipid Kinases. Journal of Biological Chemistry 2005, 280, 29053-29059, doi:10.1074/jbc.M504730200.

5. Conole, D.; Mondal, M.; Majmudar, J.D.; Tate, E.W. Recent Developments in Cell Permeable Deubiquitinating Enzyme Activity-Based Probes. Frontiers in Chemistry 2019, 0, 876, doi:10.3389/FCHEM.2019.00876.

6. Nguyen, C.; West, G.M.; Geoghegan, K.F. Emerging Methods in Chemoproteomics with Relevance to Drug Discovery. In Methods in Molecular Biology; Humana Press Inc., 2017; Vol. 1513, pp. 11-22.

7. Wang, S.; Tian, Y.; Wang, M.; Wang, M.; Sun, G.; Sun, X. Advanced Activity-Based Protein Profiling Application Strategies for Drug Development. Frontiers in Pharmacology 2018, 9, doi:10.3389/fphar.2018.00353.

8. Hershko, A.; Ciechanover, A. The Ubiquitin System for Protein Degradation. Annual Review of Biochemistry 1992, 61, 761-807, doi:10.1146/annurev.bi.61.070192.003553. 
9. Mukhopadhyay, D.; Riezman, H. Proteasome-Independent Functions of Ubiquitin in Endocytosis and Signaling. Science 2007, 315, 201-205, doi:10.1126/science.1127085.

10. Chen, R.-H.; Chen, Y.-H.; Huang, T.-Y. Ubiquitin-Mediated Regulation of Autophagy. Journal of Biomedical Science 2019 26:1 2019, 26, 1-12, doi:10.1186/S12929-019-0569-Y.

11. Harrigan, J.A.; Jacq, X.; Martin, N.M.; Jackson, S.P. Deubiquitylating Enzymes and Drug Discovery: Emerging Opportunities. Nature Reviews Drug Discovery 2018, 17, 57-78, doi:10.1038/nrd.2017.152.

12. Altun, M.; Kramer, H.B.; Willems, L.I.; McDermott, J.L.; Leach, C.A.; Goldenberg, S.J.; Kumar, K.G.S.; Konietzny, R.; Fischer, R.; Kogan, E.; et al. Activity-Based Chemical Proteomics Accelerates Inhibitor Development for Deubiquitylating Enzymes. Chemistry and Biology 2011, 18, 1401-1412, doi:10.1016/j.chembiol.2011.08.018.

13. Pinto-Fernández, A.; Davis, S.; Schofield, A.B.; Scott, H.C.; Zhang, P.; Salah, E.; Mathea, S.; Charles, P.D.; Damianou, A.; Bond, G.; et al. Comprehensive Landscape of Active Deubiquitinating Enzymes Profiled by Advanced Chemoproteomics. Frontiers in Chemistry 2019, 7, 592, doi:10.3389/fchem.2019.00592.

14. Hanpude, P.; Bhattacharya, S.; Dey, A.; life, T.M.-I.; 2015, undefined Deubiquitinating Enzymes in Cellular Signaling and Disease Regulation. Wiley Online Library 2015, 67, 544-555, doi:10.1002/iub.1402.

15. Ekkebus, R.; van Kasteren, S.I.; Kulathu, Y.; Scholten, A.; Berlin, I.; Geurink, P.P.; de Jong, A.; Goerdayal, S.; Neefjes, J.; Heck, A.J.R.; et al. On Terminal Alkynes That Can React with Active-Site Cysteine Nucleophiles in Proteases. Journal of the American Chemical Society 2013, 135, 2867-2870, doi:10.1021/ja309802n.

16. Ruiz, E.J.; Pinto-Fernandez, A.; Turnbull, A.P.; Lan, L.; Charlton, T.M.; Scott, H.C.; Damianou, A.; Vere, G.; Riising, E.M.; da Costa, C.; et al. USP28 Deletion and Small-Molecule Inhibition Destabilizes c-MYC and Elicits Regression of Squamous Cell Lung Carcinoma. eLife 2021, 10, doi:10.7554/eLife.71596.

17. Jones, H.B.L.; Heilig, R.; Fischer, R.; Kessler, B.M.; Pinto-Fernández, A. ABPP-HT - High-Throughput Activity-Based Profiling of Deubiquitylating Enzyme Inhibitors in a Cellular Context. Frontiers in Chemistry 2021, 9, doi:10.3389/fchem.2021.640105.

18. Doerr, A. DIA Mass Spectrometry. Nature Methods 2015 12:1 2014, 12, 35-35, doi:10.1038/nmeth.3234.

19. Meier, F.; Brunner, A.-D.; Frank, M.; Ha, A.; Bludau, I.; Voytik, E.; Kaspar-Schoenefeld, S.; Lubeck, M.; Raether, O.; Bache, N.; et al. DiaPASEF: Parallel Accumulation-Serial Fragmentation Combined with Data-Independent Acquisition. Nature Methods 2020 17:12 2020, 17, 1229-1236, doi:10.1038/s41592-02000998-0.

20. Demichev, V.; Messner, C.B.; Vernardis, S.I.; Lilley, K.S.; Ralser, M. DIA-NN: Neural Networks and Interference Correction Enable Deep Proteome Coverage in High Throughput. Nature Methods 2019 17:1 2019, 17, 41-44, doi:10.1038/s41592-019-0638-x.

21. Demichev, V.; Yu, F.; Teo, G.C.; Szyrwiel, L.; Rosenberger, G.A.; Decker, J.; Kaspar-Schoenefeld, S.; Lilley, K.S.; Mülleder, M.; Nesvizhskii, A.I.; et al. High Sensitivity Dia-PASEF Proteomics with DIANN and FragPipe., doi:10.1101/2021.03.08.434385.

22. Sinitcyn, P.; Hamzeiy, H.; Salinas Soto, F.; Itzhak, D.; McCarthy, F.; Wichmann, C.; Steger, M.; Ohmayer, U.; Distler, U.; Kaspar-Schoenefeld, S.; et al. MaxDIA Enables Library-Based and Library-Free DataIndependent Acquisition Proteomics. Nature Biotechnology 2021 2021, 1-11, doi:10.1038/s41587-02100968-7.

23. Tyanova, S.; Temu, T.; Cox, J. The MaxQuant Computational Platform for Mass Spectrometry-Based Shotgun Proteomics. Nature Protocols 2016 11:12 2016, 11, 2301-2319, doi:10.1038/nprot.2016.136.

24. Kong, A.T.; Leprevost, F. v; Avtonomov, D.M.; Mellacheruvu, D.; Nesvizhskii, A.I. MsFragger: Ultrafast and Comprehensive Peptide Identification in Mass Spectrometry-Based Proteomics. 2017, 14, 513, doi:10.1038/nmeth.4256.

25. Leprevost, F. da V.; Haynes, S.E.; Avtonomov, D.M.; Chang, H.-Y.; Shanmugam, A.K.; Mellacheruvu, D.; Kong, A.T.; Nesvizhskii, A.I. Philosopher: A Versatile Toolkit for Shotgun Proteomics Data Analysis. Nature methods 2020, 17, 869, doi:10.1038/S41592-020-0912-Y. 
26. Yu, F.; Haynes, S.E.; Nesvizhskii, A.I. IonQuant Enables Accurate and Sensitive Label-Free Quantification With FDR-Controlled Match-Between-Runs. Molecular \& Cellular Proteomics 2021, 20, 100077, doi:10.1016/J.MCPRO.2021.100077.

27. Michalski, A.; Cox, J.; Mann, M. More than 100,000 Detectable Peptide Species Elute in Single Shotgun Proteomics Runs but the Majority Is Inaccessible to Data-Dependent LC-MS/MS. Journal of Proteome Research 2011, 10, 1785-1793, doi:10.1021/PR101060V.

28. Turnbull, A.P.; Ioannidis, S.; Krajewski, W.W.; Pinto-Fernandez, A.; Heride, C.; Martin, A.C.L.; Tonkin, L.M.; Townsend, E.C.; Buker, S.M.; Lancia, D.R.; et al. Molecular Basis of USP7 Inhibition by Selective Small-Molecule Inhibitors. Nature 2017, 550, 481-486, doi:10.1038/nature24451.

29. Palazón-Riquelme, P.; Worboys, J.D.; Green, J.; Valera, A.; Martín-Sánchez, F.; Pellegrini, C.; Brough, D.; López-Castejón, G. USP7 and USP47 Deubiquitinases Regulate NLRP3 Inflammasome Activation. EMBO reports 2018, 19, e44766, doi:10.15252/embr.201744766.

30. Ruiz, E.J.; Pinto-Fernandez, A.; Turnbull, A.P.; Lan, L.; Charlton, T.M.; Scott, H.C.; Damianou, A.; Vere, G.; Riising, E.M.; da Costa, C.; et al. USP28 Deletion and Small-Molecule Inhibition Destabilizes c-MYC and Elicits Regression of Squamous Cell Lung Carcinoma. eLife 2021, 10, doi:10.7554/eLife.71596.

31. Chapman, J.D.; Goodlett, D.R.; Masselon, C.D. Multiplexed and Data-Independent Tandem Mass Spectrometry for Global Proteome Profiling. Mass Spectrometry Reviews 2014, 33, 452-470, doi:10.1002/MAS.21400.

32. Bekker-Jensen, D.B.; Bernhardt, O.M.; Hogrebe, A.; Martinez-Val, A.; Verbeke, L.; Gandhi, T.; Kelstrup, C.D.; Reiter, L.; Olsen, J. v. Rapid and Site-Specific Deep Phosphoproteome Profiling by DataIndependent Acquisition without the Need for Spectral Libraries. Nature Communications 2020, 11, 787, doi:10.1038/s41467-020-14609-1.

33. Steger, M.; Demichev, V.; Backman, M.; Ohmayer, U.; Ihmor, P.; Müller, S.; Ralser, M.; Daub, H. TimeResolved in Vivo Ubiquitinome Profiling by DIA-MS Reveals USP7 Targets on a Proteome-Wide Scale. Nature Communications 2021, 12, 5399, doi:10.1038/s41467-021-25454-1.

34. Hansen, F.M.; Tanzer, M.C.; Brüning, F.; Bludau, I.; Stafford, C.; Schulman, B.A.; Robles, M.S.; Karayel, O.; Mann, M. Data-Independent Acquisition Method for Ubiquitinome Analysis Reveals Regulation of Circadian Biology. Nature Communications 2021, 12, 254, doi:10.1038/s41467-020-20509-1.

35. Colland, F.; Formstecher, E.; Jacq, X.; Reverdy, C.; Planquette, C.; Conrath, S.; Trouplin, V.; Bianchi, J.; Aushev, V.N.; Camonis, J.; et al. Small-Molecule Inhibitor of USP7/HAUSP Ubiquitin Protease Stabilizes and Activates P53 in Cells. Molecular Cancer Therapeutics 2009, 8, 2286-2295, doi:10.1158/15357163.MCT-09-0097.

36. Borodovsky, A.; Ovaa, H.; Kolli, N.; Gan-Erdene, T.; Wilkinson, K.D.; Ploegh, H.L.; Kessler, B.M. Chemistry-Based Functional Proteomics Reveals Novel Members of the Deubiquitinating Enzyme Family. Chemistry and Biology 2002, 9, 1149-1159, doi:10.1016/S1074-5521(02)00248-X.

37. Bache, N.; Geyer, P.E.; Bekker-Jensen, D.B.; Hoerning, O.; Falkenby, L.; Treit, P. v.; Doll, S.; Paron, I.; Müller, J.B.; Meier, F.; et al. A Novel LC System Embeds Analytes in Pre-Formed Gradients for Rapid, Ultra-Robust Proteomics. Molecular \& Cellular Proteomics 2018, 17, 2284-2296, doi:10.1074/mcp.TIR118.000853.

38. Lex, A.; Gehlenborg, N.; Strobelt, H.; Vuillemot, R.; Pfister, H. UpSet: Visualization of Intersecting Sets. IEEE Transactions on Visualization and Computer Graphics 2014, 20, 1983-1992, doi:10.1109/TVCG.2014.2346248. 


\section{Scheme 1}
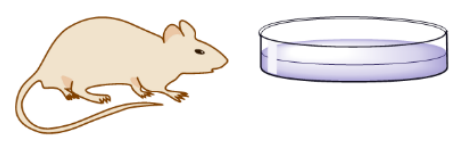

A Protein extraction

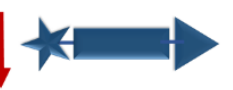

B Probe incubation

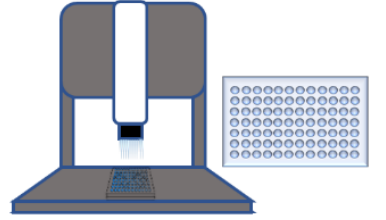

C IAP-MS sample preparation

High-throughput workflow 100 samples per day

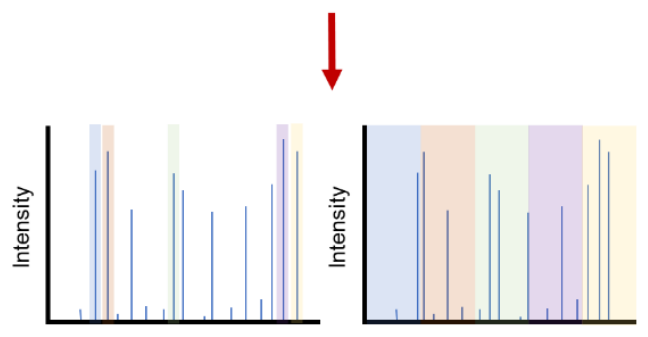

D LC-MS/MS: DDA and DIA

$\mathrm{m} / \mathrm{z}$

$\mathrm{m} / \mathrm{z}$ (Evosep/timsTOF)

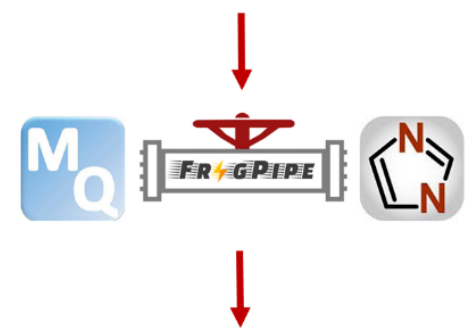

E Search engines:

- Fragpipe (DDA)

- Maxquant (DDA)

- DIA-NN (DIA)

- MaxDIA (DIA)

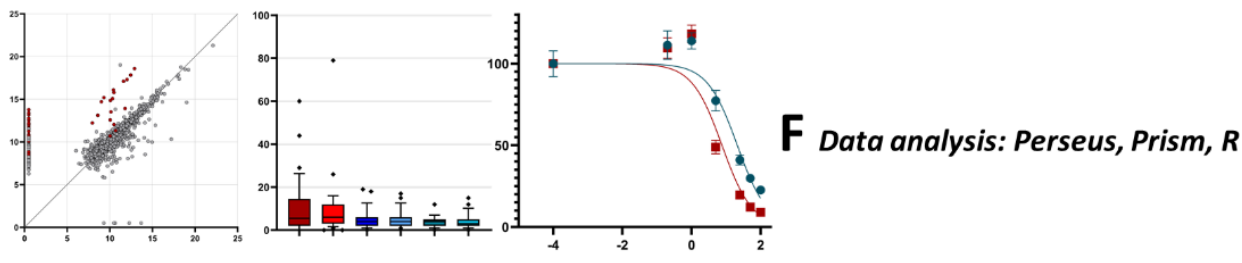


bioRxiv preprint doi: https://doi org/10.1101/2022 02 18.480987 this version posted February 21, 2022 The copyright holder for this preprint (which was not certified by peer review) is the author/funder, who has granted bioRxiv a license to display the preprint in perpetuity. It is made available under aCC-BY-ND 4.0 International license.

\section{Figure 1}

A

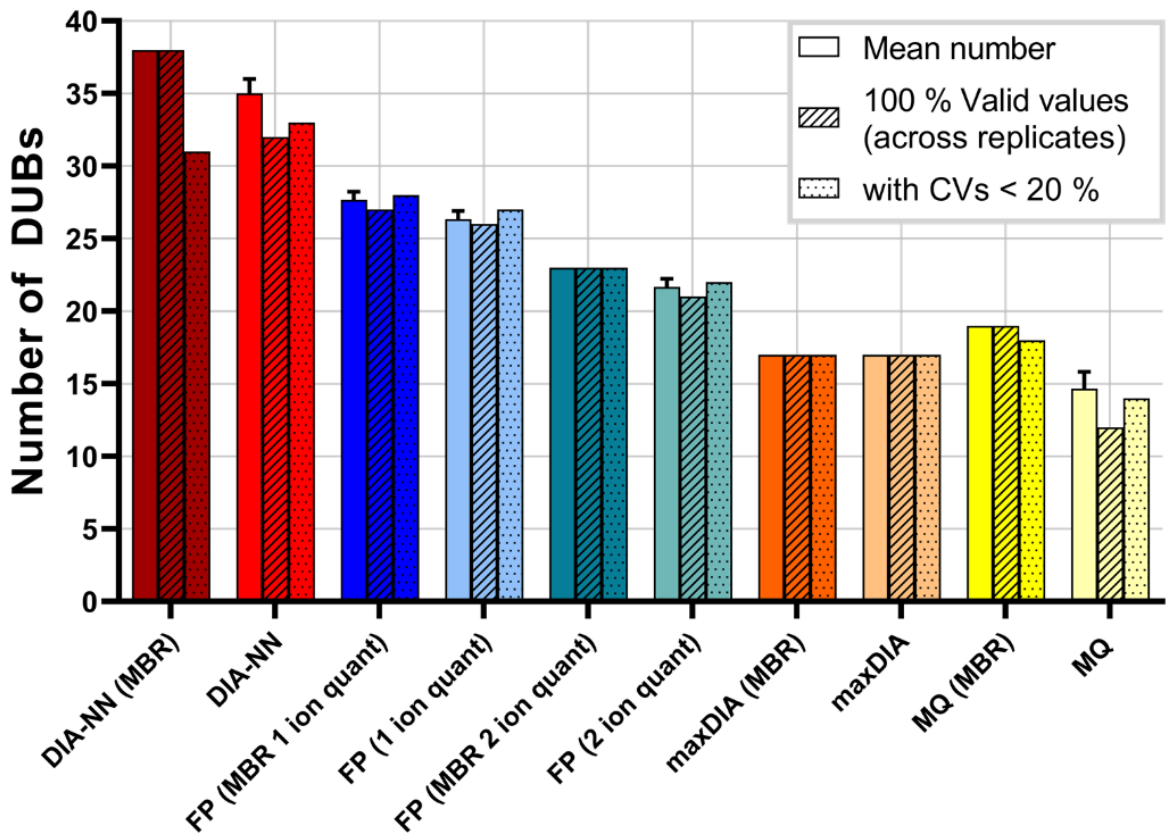

B

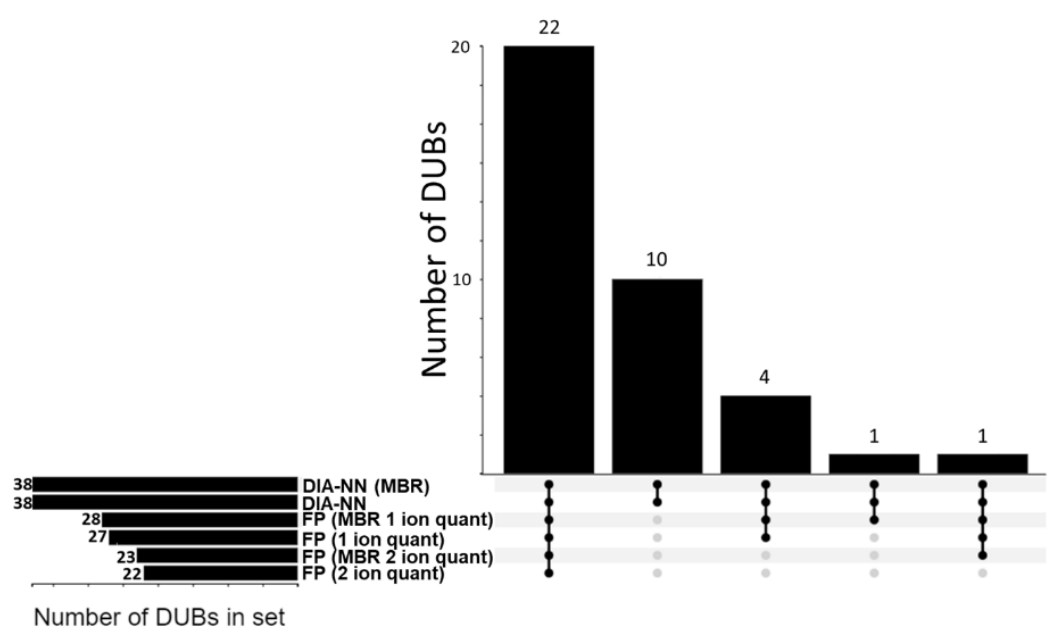

C

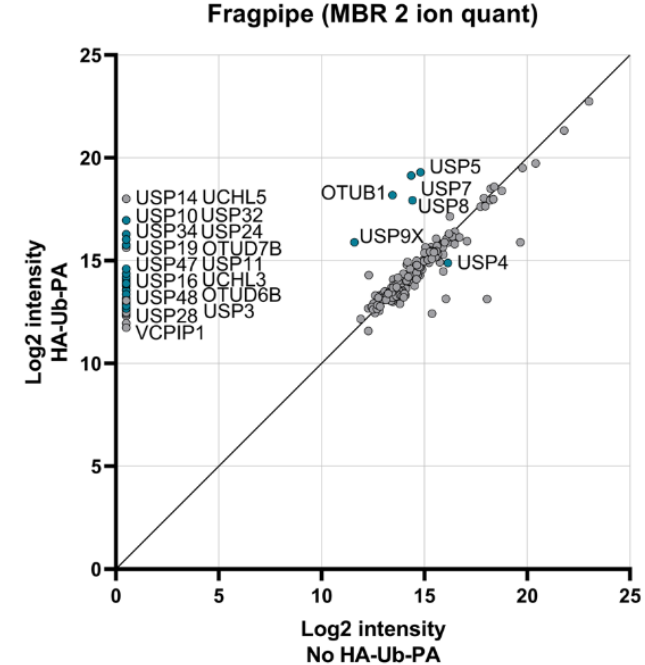

D

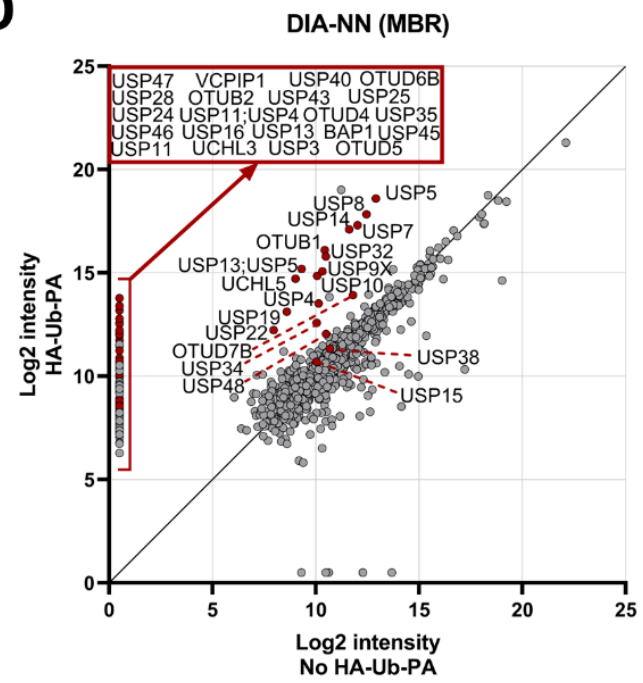


bioRxiv preprint doi: https:/doi.org/10.1101/2022.02 18.480987: this version posted February 21, 2022. The copyright holder for this preprint (which was not certified by peer review) is the author/funder, who has granted bioRxiv a license to display the preprint in perpetuity. It is made available under aCC-BY-ND 4.0 International license.

\section{Figure 2}

A
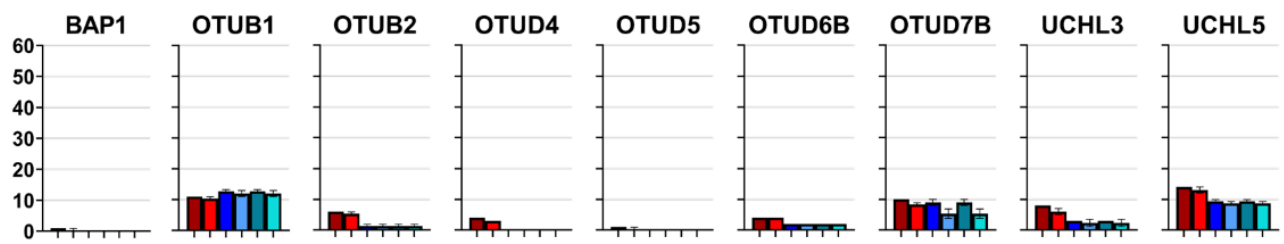

USP3
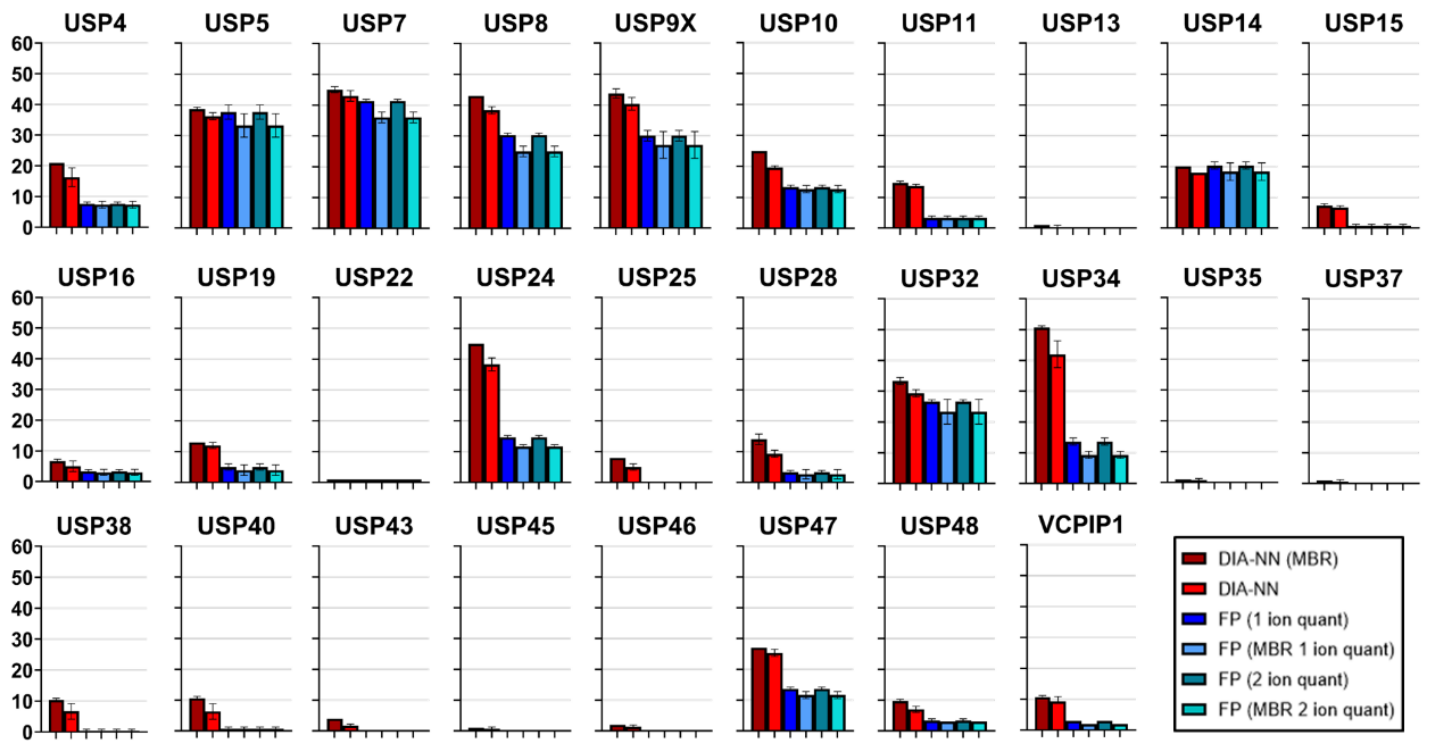

B

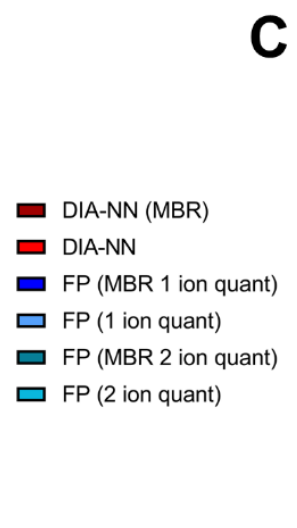

C
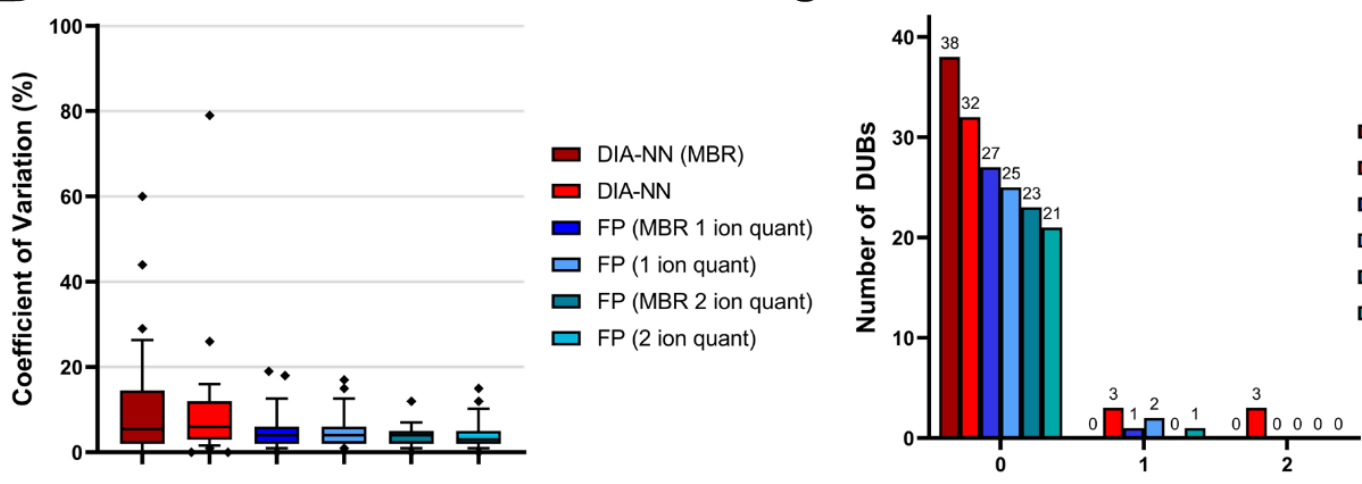

$\square$ DIA-NN (MBR)

$\square$ DIA-NN

$\square$ FP (MBR 1 ion quant)

$\square$ FP (1 ion quant)

$\square$ FP (MBR 2 ion quant)

$\square$ FP (2 ion quant)

Number of missing values 


\section{Figure 3}

A

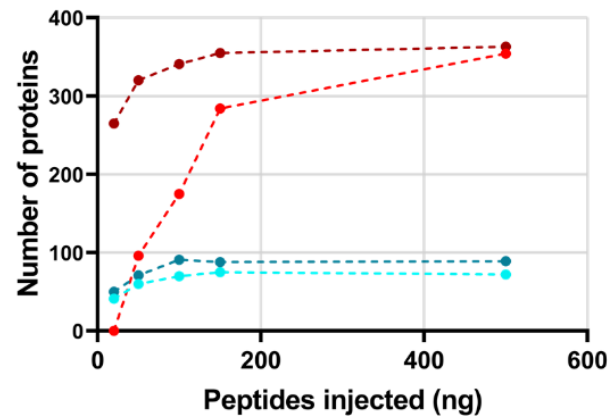

C

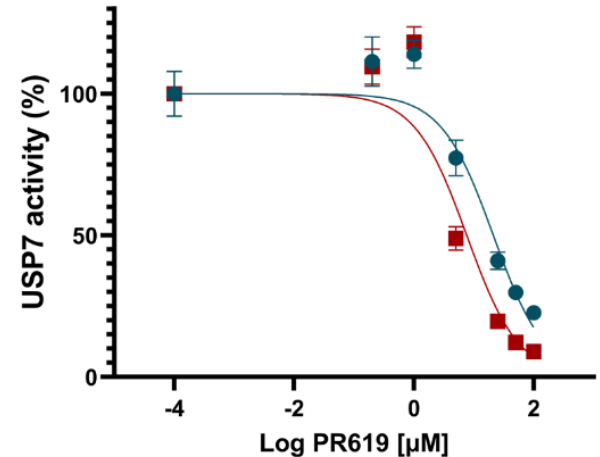

$\mathbf{E}$

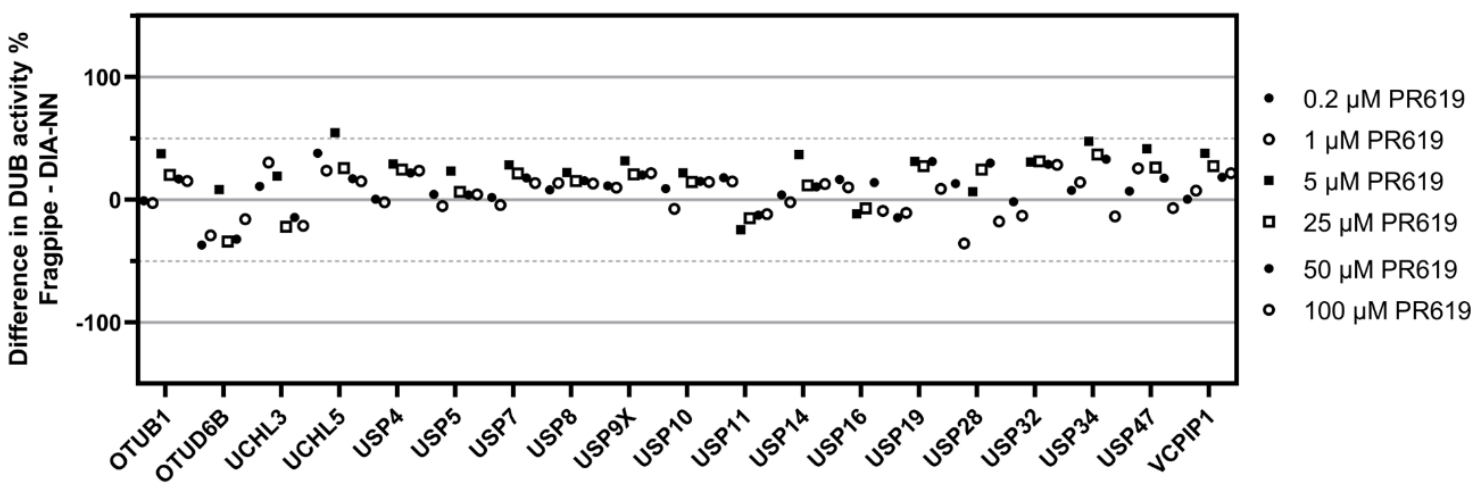

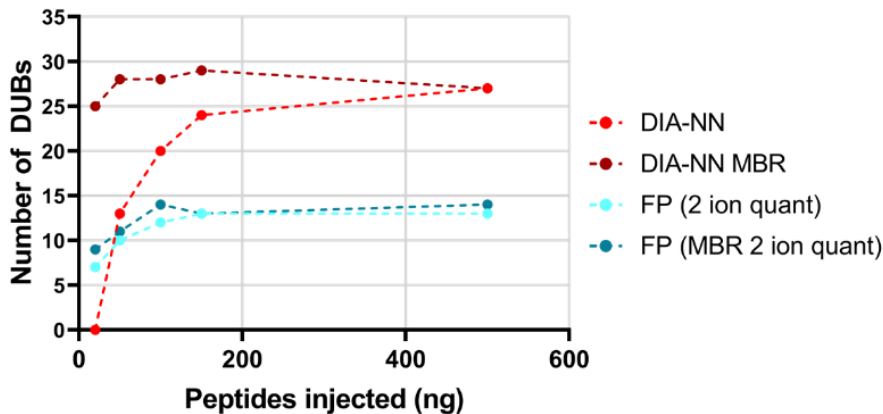

D

USP7 with PR619

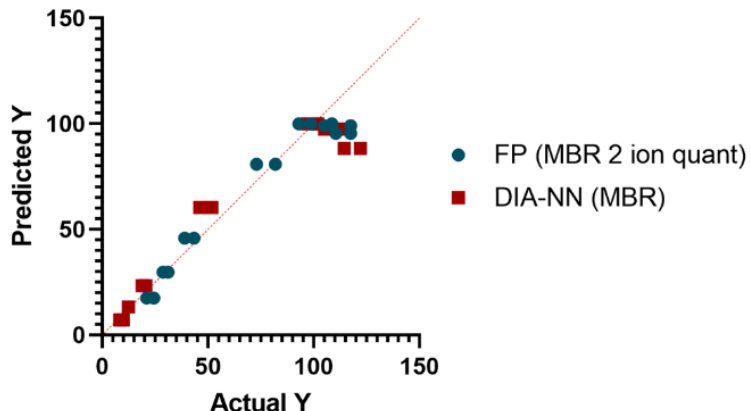


bioRxiv preprint doi: https://doi.org/10.1101/2022.02 18.480987; this version posted February 21, 2022. The copyright holder for this preprint (which was not certified by peer review) is the author/funder, who has granted bioRxiv a license to display the preprint in perpetuity. It is made available under aCC-BY-ND 4.0 International license.

\section{Figure 4}

A

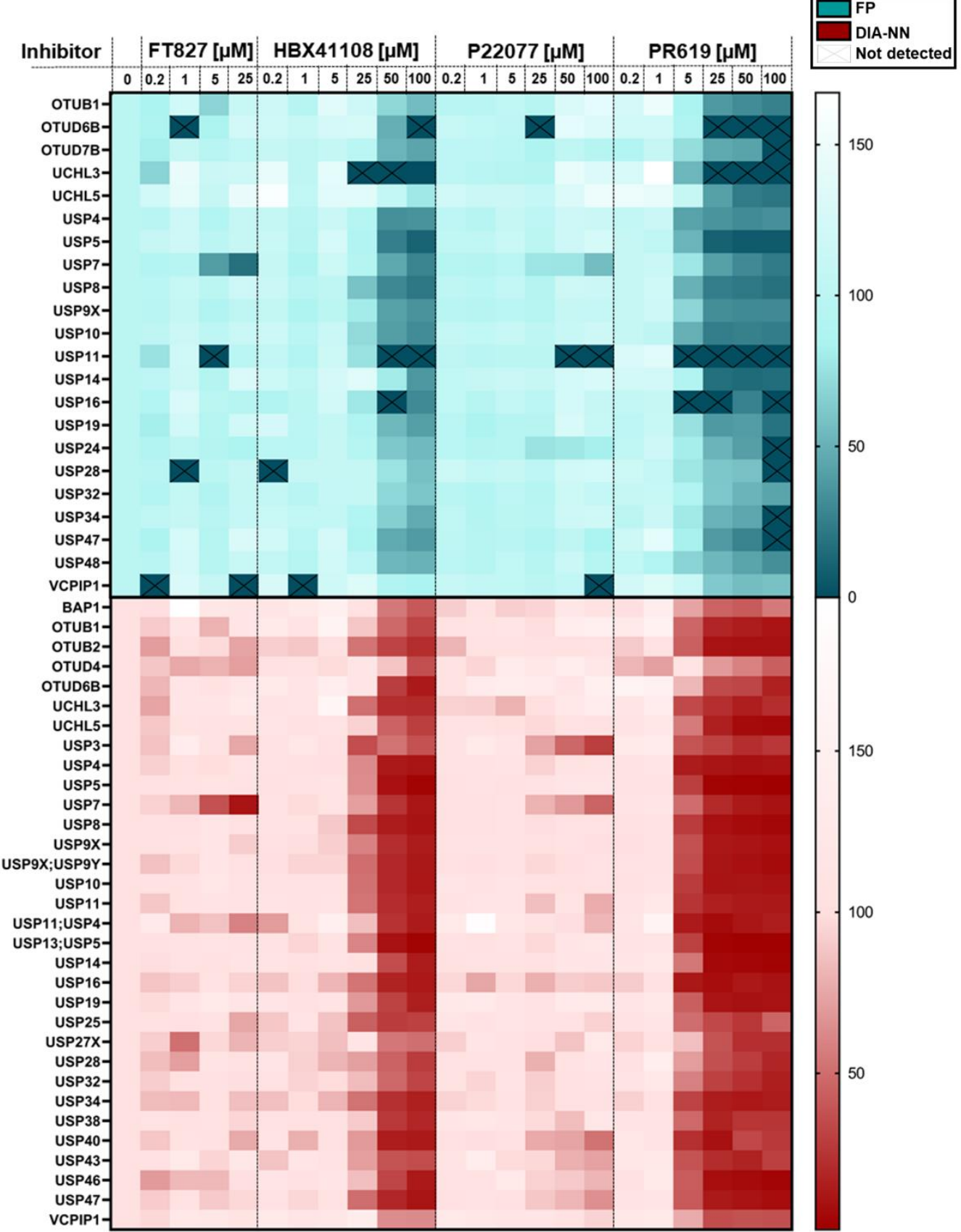

B

\begin{tabular}{|c|c|c|c|c|c|c|c|c|c|}
\hline & \multicolumn{3}{|c|}{ Fragpipe } & \multicolumn{3}{|c|}{ DIA-NN } & \multicolumn{3}{|c|}{ Maxquant (Previously reported [17]) } \\
\hline & $\begin{array}{l}\text { USP7 } \\
\text { IC50 } \\
(\mu \mathrm{M})\end{array}$ & $\begin{array}{c}95 \% \\
\text { Confidence } \\
\text { interval }\end{array}$ & R squared & $\begin{array}{l}\text { USP7 } \\
\text { IC50 } \\
\text { ( } \mu \mathrm{M})\end{array}$ & $\begin{array}{c}95 \% \\
\text { Confidence } \\
\text { interval }\end{array}$ & R squared & $\begin{array}{l}\text { USP7 } \\
\text { IC50 } \\
(\mu \mathrm{M})\end{array}$ & $\begin{array}{c}95 \% \\
\text { Confidence } \\
\text { interval }\end{array}$ & R squared \\
\hline FT827 & 4.6 & $2.5-8.4$ & 0.94 & 3.3 & $2.7-4.1$ & 0.99 & 2.1 & $1.5-2.8$ & 0.99 \\
\hline PR619 & 21 & $14.2-30.9$ & 0.93 & 7.6 & $4.5-12.9$ & 0.92 & 7 & $3.6-13.2$ & 0.95 \\
\hline HBX41108 & 64.3 & $31.5-142.3$ & 0.79 & 32.9 & $17.5-61.1$ & 0.89 & 37.2 & $19.2-70.7$ & 0.87 \\
\hline P22077 & 127 & $86.7-196$ & 0.85 & 99.1 & 75.3 to 133.2 & 0.95 & 62.8 & $42.8-93.9$ & 0.95 \\
\hline
\end{tabular}


bioRxiv preprint doi: https://doi.org/10.1101/2022.02 18.480987; this version posted February 21, 2022. The copyright holder for this preprint (which was not certified by peer review) is the author/funder, who has granted bioRxiv a license to display the preprint in perpetuity. It is made available under aCC-BY-ND 4.0 International license.

\section{Scheme 2}

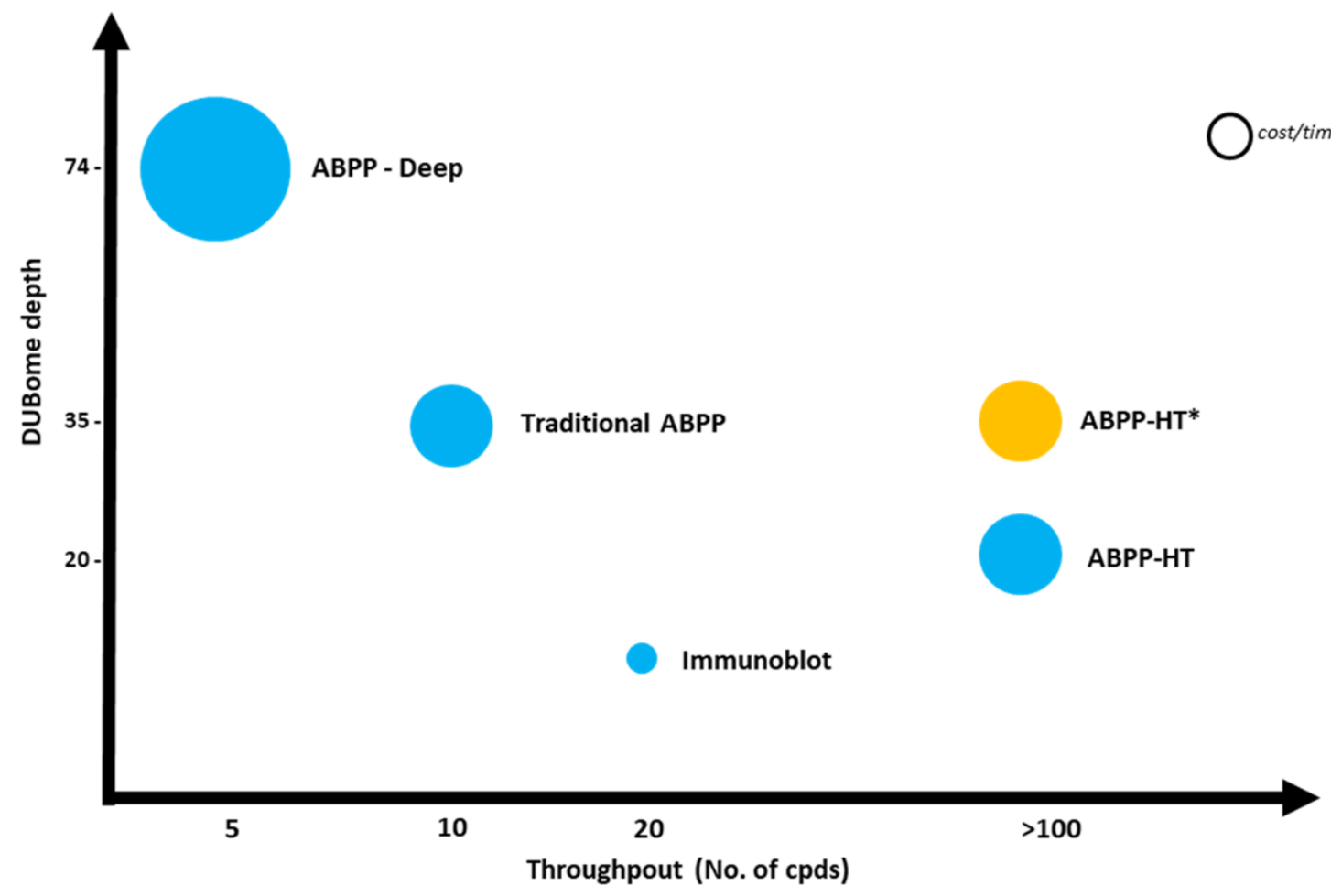




\section{Figure S1}

A

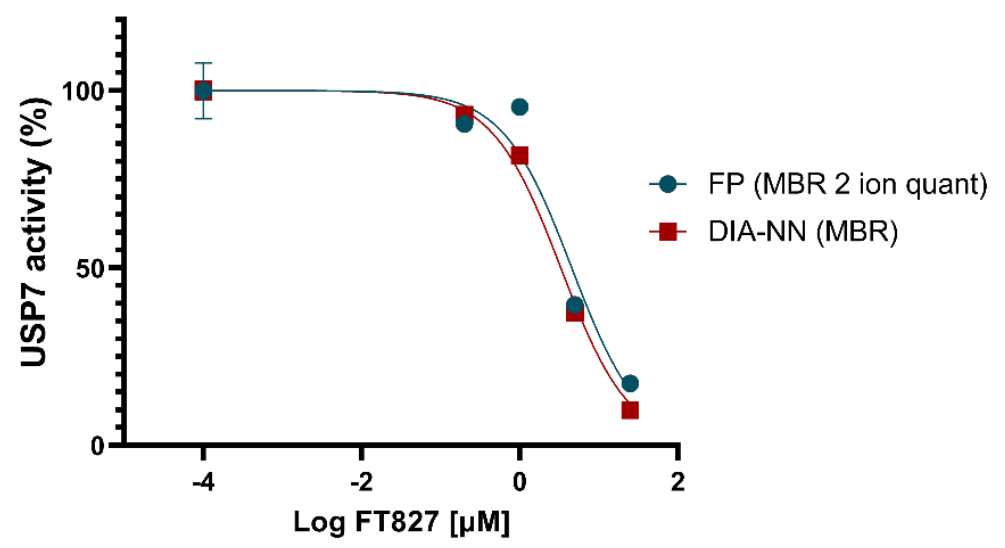

B

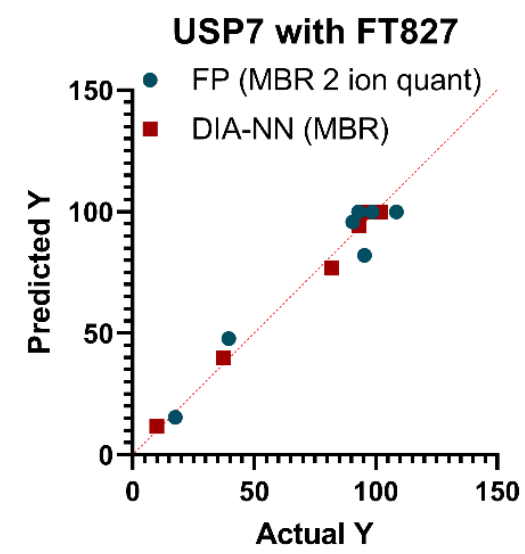

C

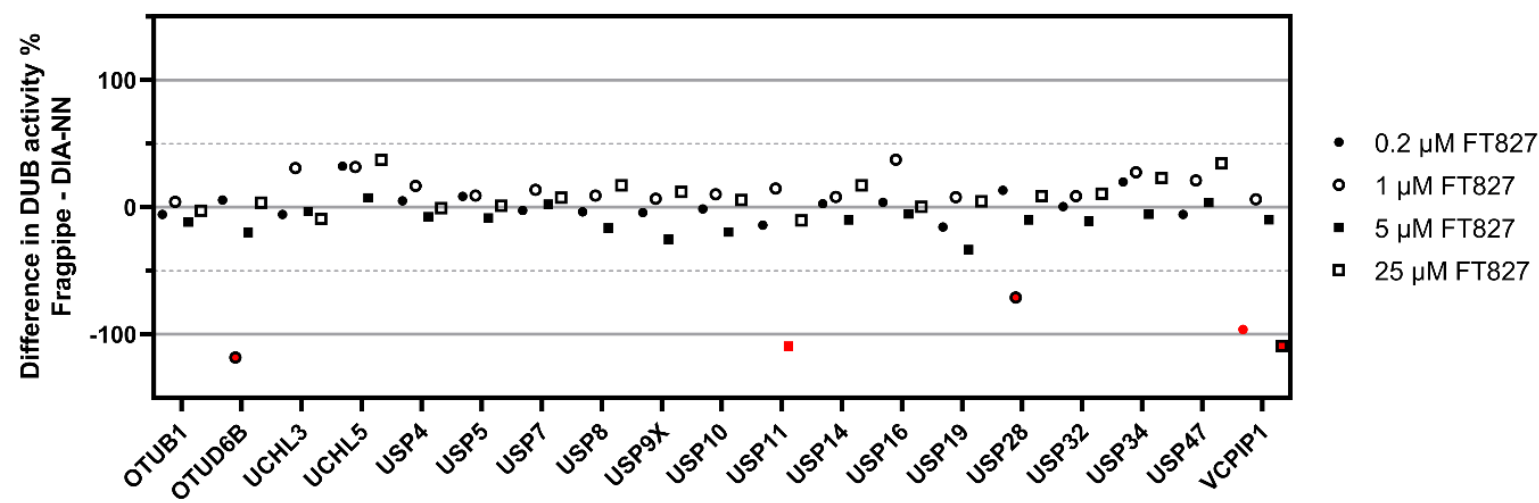

D

USP7 with PR619

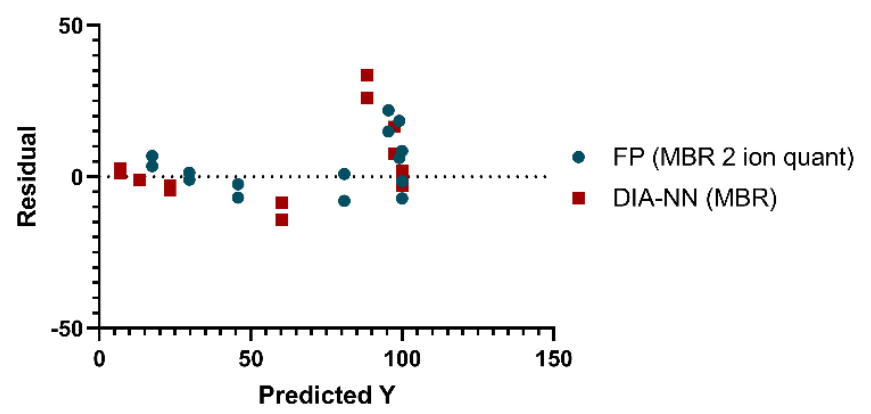

$\mathbf{E}$

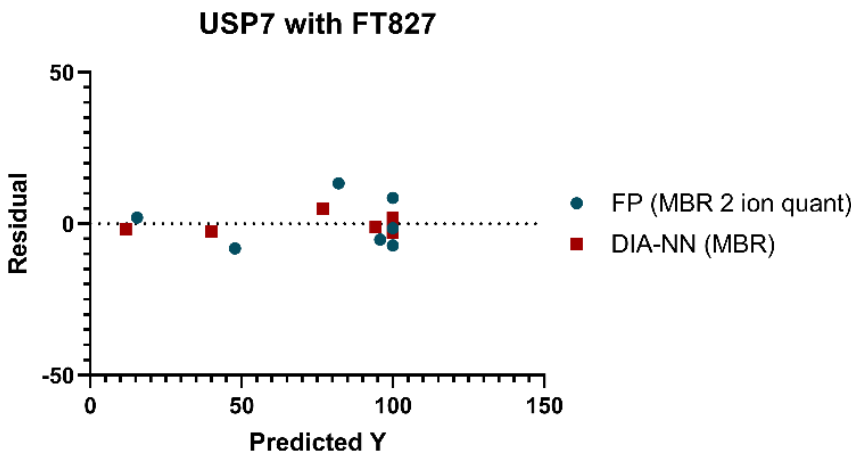

Supplementary Figure 1. A) USP7 IC 50 curve showing reduction of USP7 intensity with increasing FT827 concentrations, collected as either DIA data analysed with DIA-NN MBR* or DDA data Fragpipe data 2 ion quant $\mathrm{MBR}^{*}$ fit to $\mathrm{Y}=100 /\left(1+10^{\wedge}\left(\left(\mathrm{X}-\log \mid \mathrm{C}_{50}\right)\right)\right)$. ${ }^{*} \mathrm{MBR}$ as part of a larger dataset with other inhibitors. B) Predicted vs actual $Y$ values of the $I_{50}$ curve shown in $A$. C. Difference plot of common DUBs identified by both DIA-NN MBR and Fragpipe 2 ion quant MBR with DIA-NN \% activity subtracted from Fragpipe \% activity at various FT827 concentrations. Values in red are attributed to missing 
bioRxiv preprint doi: https://doi.org/10.1101/2022.02.18.480987; this version posted February 21, 2022. The copyright holder for this preprint (which was not certified by peer review) is the author/funder, who has granted bioRxiv a license to display the preprint in perpetuity. It is made available under aCC-BY-ND 4.0 International license.

values and not as a result of inhibition differences due to their reduction not occurring in a concentration dependent manner with increasing inhibitor concentration. D) Residual vs predicted $Y$ for USP7 with PR619 IC 50 fit. E) Residual vs predicted Y for USP7 with FT827 IC 50 fit. 


\section{Supplementary Table 1}

\begin{tabular}{|c|c|c|c|}
\hline Start $\mathrm{m} / \mathrm{z}$ & End $m / z$ & Start $1 / \mathrm{k} 0$ & End $1 / \mathrm{k} 0$ \\
\hline 400 & 425 & 0.6 & 0.834 \\
\hline 425 & 450 & 0.6 & 0.858 \\
\hline 450 & 475 & 0.6 & 0.883 \\
\hline 475 & 500 & 0.6 & 0.908 \\
\hline 500 & 525 & 0.6 & 0.932 \\
\hline 525 & 550 & 0.6 & 0.957 \\
\hline 550 & 575 & 0.6 & 0.982 \\
\hline 575 & 600 & 0.6 & 1.005 \\
\hline 600 & 625 & 0.835 & 1.031 \\
\hline 625 & 650 & 0.858 & 1.055 \\
\hline 650 & 675 & 0.884 & 1.079 \\
\hline 675 & 700 & 0.909 & 1.105 \\
\hline 700 & 725 & 0.934 & 1.129 \\
\hline 725 & 750 & 0.958 & 1.153 \\
\hline 750 & 775 & 0.983 & 1.179 \\
\hline 775 & 800 & 1.006 & 1.202 \\
\hline 800 & 825 & 1.032 & 1.638 \\
\hline 825 & 850 & 1.056 & 1.638 \\
\hline 850 & 875 & 1.08 & 1.638 \\
\hline 875 & 900 & 1.106 & 1.638 \\
\hline 900 & 925 & 1.13 & 1.638 \\
\hline 925 & 950 & 1.154 & 1.638 \\
\hline 950 & 975 & 1.18 & 1.638 \\
\hline 975 & 1000 & 1.204 & 1.638 \\
\hline
\end{tabular}

\section{Supplementary Table 1}

DIA-PASEF windows for data in Figures $1,2,3 \mathrm{C}-\mathrm{E}+4$. 


\section{Supplementary Table 2}

\begin{tabular}{|c|c|c|c|}
\hline Start $\mathrm{m} / \mathrm{z}$ & End $m / z$ & Start $1 / \mathrm{k} 0$ & End $1 / \mathrm{k} 0$ \\
\hline 475 & 500 & 0.85 & 0.89 \\
\hline 500 & 525 & 0.85 & 0.91 \\
\hline 525 & 550 & 0.85 & 0.93 \\
\hline 550 & 575 & 0.85 & 0.95 \\
\hline 575 & 600 & 0.85 & 0.97 \\
\hline 600 & 625 & 0.85 & 0.99 \\
\hline 625 & 650 & 0.85 & 1.01 \\
\hline 650 & 675 & 0.87 & 1.03 \\
\hline 675 & 700 & 0.89 & 1.04 \\
\hline 700 & 725 & 0.91 & 1.06 \\
\hline 725 & 750 & 0.93 & 1.08 \\
\hline 750 & 775 & 0.95 & 1.1 \\
\hline 775 & 800 & 0.97 & 1.12 \\
\hline 800 & 825 & 0.99 & 1.14 \\
\hline 825 & 850 & 1.01 & 1.16 \\
\hline 850 & 875 & 1.03 & 1.18 \\
\hline 875 & 900 & 1.05 & 1.2 \\
\hline 900 & 925 & 1.07 & 1.22 \\
\hline 925 & 950 & 1.08 & 1.24 \\
\hline 950 & 975 & 1.1 & 1.26 \\
\hline 975 & 1000 & 1.12 & 1.27 \\
\hline
\end{tabular}

\section{Supplementary Table 2}

DIA-PASEF windows for data in Figures 3A + B. 